\title{
Correspondence
}

\section{Balanced Multiwavelets Theory and Design}

\author{
Jérôme Lebrun and Martin Vetterli
}

\begin{abstract}
This correspondence deals with multiwavelets, which are a recent generalization of wavelets in the context of time-varying filter banks and with their applications to signal processing and especially compression. By their inherent structure, multiwavelets are fit for processing multichannel signals. This is the main issue in which we will be interested here. The outline of the correspondence is as follows. First, we will review material on multiwavelets and their links with multifilter banks and, especially, time-varying filter banks. Then, we will have a close look at the problems encountered when using multiwavelets in applications, and we will propose new solutions for the design of multiwavelets filter banks by introducing the so-called balanced multiwavelets.
\end{abstract}

Index Terms-Balancing, multifilter, multiwavelet, prefiltering, timevarying filter bank.

\section{INTRODUCTION}

Wavelet constructions from iterated filter banks, as pioneered by Daubechies, have become a standard way to derive orthogonal and biorthogonal wavelet bases. The underlying filter banks are well studied, and thus, the design procedure is well understood. By the structure of the problem, certain issues are ruled out. The impossibility of constructing orthogonal FIR linear-phase filter banks implies that there is no orthogonal wavelet with compact support and symmetry. Nevertheless, by relaxing the requirement of time invariance, it is easy to see that new solutions are possible. As mentioned in [14], such filter banks are closely related to some matrix 2-scale equations leading to multiwavelets. First, we will recall the basics about multiwavelets. Then, we will link this to multifilter banks and time-varying filter banks. Then, we will define under what conditions we can apply systems based on multiwavelets to onedimensional (1-D) signals in a simple way. That means we will give some natural and simple conditions that should help in the design of new multiwavelets for signal processing. Finally, we will provide some tools in order to construct multiwavelets with the required properties: the so-called balanced multiwavelets.

\section{MultiwaVELETS}

Generalizing the wavelet case, one can allow a multiresolution analysis $\left\{V_{n}\right\}_{n}$ of $L^{2}(\mathbb{R})$ to be generated by a finite number of scaling functions $\phi_{0}(t), \phi_{1}(t), \cdots, \phi_{r-1}(t)$ and their integer translates. Then, the multiscaling function $\phi(t):=\left[\phi_{0}(t), \cdots, \phi_{r-1}(t)\right]^{\top}$

Manuscript received February 15, 1997; revised November 30, 1997. This work was supported in part by the National Science Foundation under Grant MIP-93-21302 and by the Swiss National Science Foundation under Grant 2100-043136.95/1. The associate editor coordinating the review of this paper and approving it for publication was Prof. Ali N. Akansu.

J. Lebrun is with the Department of Electrical Engineering, Swiss Federal Institute of Technology, Lausanne, Switzerland (e-mail: lebrun@de.epfl.ch).

M. Vetterli is with the Department of Electrical Engineering, Swiss Federal Institute of Technology, Lausanne, Switzerland, and with the Department of Electrical Engineering and Computer Science, University of California, Berkeley, CA 94720 USA (e-mail: vetterli@de.epfl.ch).

Publisher Item Identifier S 1053-587X(98)02508-2. verifies a two-scale equation

$$
\underline{\phi}(t)=\sum_{k} \mathbf{M}[k] \underline{\phi}(2 t-k)
$$

where, now, $\{\mathbf{M}[k]\}_{k}$ is a sequence of $r \times r$ matrices of real coefficients. The multiresolution analysis structure gives $V_{1}=$ $V_{0} \oplus W_{0}$, where $W_{0}$ is the orthogonal complement of $V_{0}$ in $V_{1}$. We can construct an orthonormal basis of $W_{0}$ generated by $\psi_{0}(t), \psi_{1}(t), \cdots, \psi_{r-1}(t)$ and their integer translates with $\underline{\psi}(t):=\left[\psi_{0}(t), \cdots, \psi_{r-1}(t)\right]^{\top}$ derived by

$$
\underline{\psi}(t):=\sum_{k} \mathbf{N}[k] \underline{\phi}(2 t-k)
$$

where $\{\mathbf{N}[k]\}_{k}$ is a sequence of $r \times r$ matrices of real coefficients obtained by completion of $\{\mathbf{M}[k]\}_{k}$ (see [7]). Introducing the refinement masks $\mathbf{M}(z):=\frac{1}{2} \sum_{n} \mathbf{M}[n] z^{-n}$ and $\mathbf{N}(z):=$ $\frac{1}{2} \sum_{n} \mathbf{N}[n] z^{-n}$, (1) and (2) translate in the Fourier domain into

$$
\Phi(2 \omega)=\mathbf{M}\left(e^{j \omega}\right) \Phi(\omega) \text { and } \Psi(2 \omega)=\mathbf{N}\left(e^{j \omega}\right) \Phi(\omega) .
$$

We can then derive the behavior of the multiscaling function by iterating the first product above. If this iterated matrix product converges, we get in the limit

$$
\Phi(\omega)=\mathbf{M}_{\infty}(\omega) \Phi(0)=\prod_{i=1}^{\infty} \mathbf{M}\left[e^{j\left(\omega / 2^{i}\right)}\right] \Phi(0) .
$$

For simplicity and without loss of generality, we will now on concentrate on the case $r=2$. Furthermore, we will assume that the sequences $\{\mathbf{M}[k]\}_{k}$ and $\{\mathbf{N}[k]\}_{k}$ are finite and, thus, that $\phi(t)$ and $\underline{\psi}(t)$ have compact support. We then recall some result obtained in [2] and [14] about the convergence of the iterated matrix product $\mathbf{M}_{\infty}(\omega)$. For $\mathbf{M}(z)$ satisfying a matrix Smith-Barnwell orthogonality condition

$$
\mathbf{M}(z) \mathbf{M}^{\top}\left(z^{-1}\right)+\mathbf{M}(-z) \mathbf{M}^{\top}\left(-z^{-1}\right)=\mathbf{I}
$$

a necessary condition for uniform convergence of the iterated product to a scaling matrix $\mathbf{M}_{\infty}(\omega)$ such that $\mathbf{M}_{\infty}(0)$ is nonzero and bounded is either

1) $\mathbf{M}(1)=\mathbf{I}, \mathbf{M}(-1)=\mathbf{0}$ [note that $\mathbf{M}_{\infty}(\omega)$ has rank 2];

2) $\mathbf{M}(1)$ has eigenvalue $\lambda_{0}(1)=1$ and $\left|\lambda_{1}(1)\right|<1$, and $\mathbf{M}(-1)$ has rank 1 and satisfies $\mathbf{r}_{0} \mathbf{M}(-1)=\mathbf{0}$, where $\mathbf{r}_{0}$ is a left eigenvector of $\mathbf{M}(1)$ for the eigenvalue 1 [note that $\mathbf{M}_{\infty}(\omega)$ has then rank 1].

Now, assuming (5) and 1) or 2), the scaling functions and their integer translates form an orthonormal basis of $V_{0}$. Thus, for $s(t) \in V_{0}$, we have

$$
s(t)=\sum_{n} \boldsymbol{s}_{0}^{\top}[n] \underline{\phi}(t-n)
$$

and then, from $V_{0}=V_{-1} \oplus W_{-1}$, we get

$$
s(t)=\sum_{n} \boldsymbol{s}_{-1}^{\top}[n] \underline{\phi}\left(\frac{t}{2}-n\right)+\boldsymbol{d}_{-1}^{\top}[n] \underline{\psi}\left(\frac{t}{2}-n\right)
$$

and we have the well-known relations between the coefficients at the analysis step

$$
\begin{aligned}
& \boldsymbol{s}_{-1}[n]=\sum_{k} \mathbf{M}[k-2 n] \boldsymbol{s}_{0}[k] \\
& \boldsymbol{d}_{-1}[n]=\sum_{k} \mathbf{N}[k-2 n] \boldsymbol{s}_{0}[k]
\end{aligned}
$$




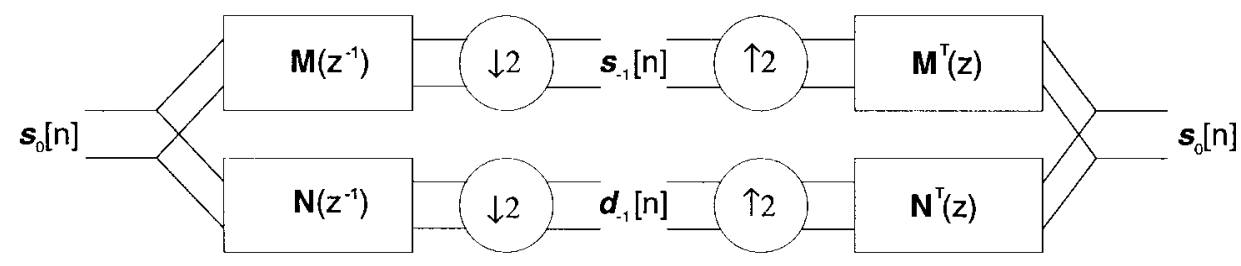

Fig. 1. Lossless orthogonal multiwavelet filter bank for $r=2$.

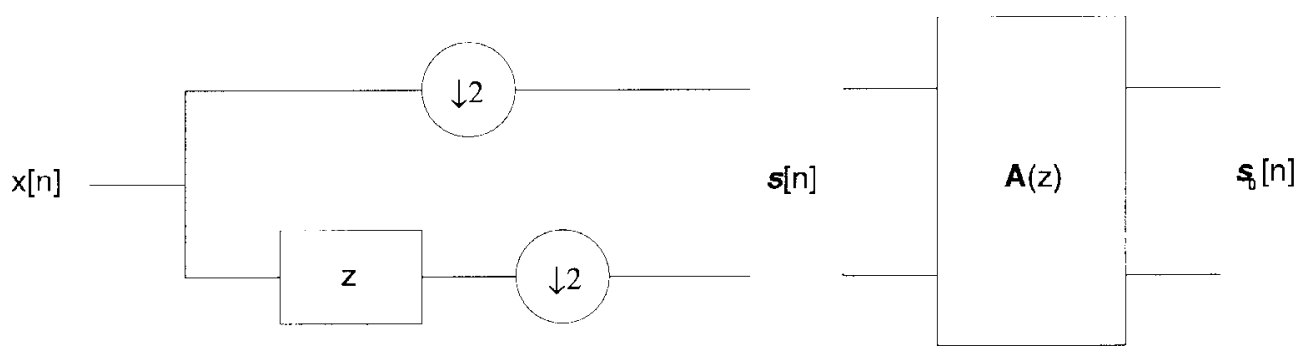

(a)
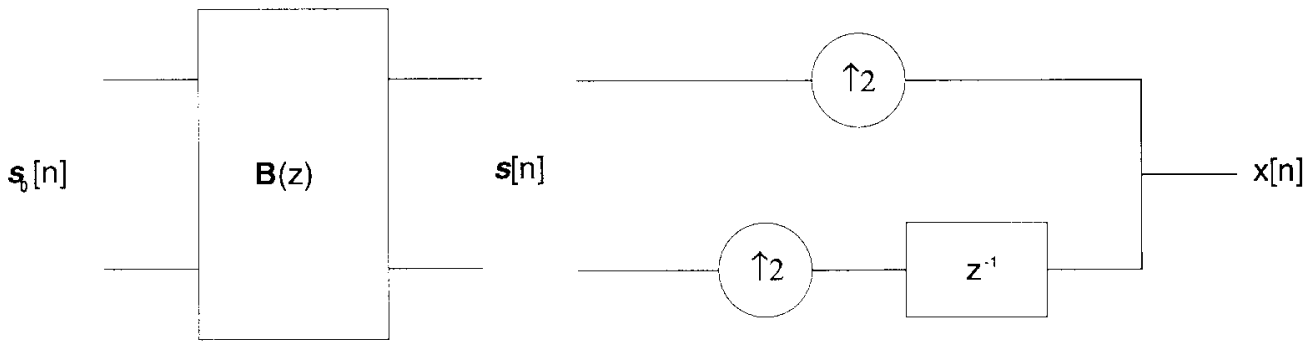

(b)

Fig. 2. Vectorization and pre/post filtering steps for a multiwavelet filter bank.

and for the synthesis, we get

$$
\boldsymbol{s}_{0}[n]=\sum_{k} \mathbf{M}^{\top}[n-2 k] \boldsymbol{s}_{-1}[k]+\mathbf{N}^{\top}[n-2 k] \boldsymbol{d}_{-1}[k] .
$$

These relations enable us to construct a multi-input multi-output (MIMO) filter bank (which is abbrieviated multifilter bank), as seen in Fig. 1.

\section{VECTORIZATION AND PREFILTERING}

We already noticed that a multiwavelet filter bank is fundamentally a MIMO system. In case of a 1-D signal, it then requires vectorization of this input signal to produce an input signal which is twodimensional (2-D). A simple way to do that is to split a 1-D signal into its polyphase components (Fig. 2). Introducing

$$
\left[\begin{array}{l}
m_{0}(z) \\
m_{1}(z)
\end{array}\right]:=\mathbf{M}\left(z^{2}\right)\left[\begin{array}{c}
1 \\
z^{-1}
\end{array}\right]
$$

and in the same way $n_{0}(z)$ and $n_{1}(z)$, the system can then be seen as a four-channel time-varying filter bank (Fig. 3). If the components $m_{0}(z)$ and $m_{1}(z)$ of the lowpass branch have different spectral behavior, e.g., lowpass behavior for one and highpass for the other, it then leads to unbalanced channels that complicate the vectorization. In that case, the polyphase method of vectorization leads to a mixing of the coarse resolution and details coefficients creating strong oscillations in the signal reconstructed from the coarse resolution only (Fig. 4). This problem is crucial. One of the important issues with wavelets in subband coding is the behavior of truncated series, i.e., the robustness to truncation of the details subbands. Thus, one expect some class of smooth signals to be well reproduced using only the coarse resolution coefficients, i.e., one expects these signals to be eigensignals of the lowpass branch and cancelled by the highpass. In the orthonormal case, defining the band-Toeplitz matrix corresponding to the lowpass analysis

$$
\mathbf{L}:=\left[\begin{array}{llllll}
\cdots & & & & & \\
& \mathbf{M}[0] & \mathbf{M}[1] & \mathbf{M}[2] & \mathbf{M}[3] & \ldots \\
& & & \mathbf{M}[0] & \mathbf{M}[1] & \ldots \\
& & & & & \cdots
\end{array}\right]
$$

we then require $\mathbf{u}_{1}:=[\cdots, 1,1,1,1, \cdots]^{\top}$ to be preserved by the lowpass synthesis operator $\mathbf{L}^{\top}$, i.e.,

$$
\mathbf{L}^{\top}[\cdots, 1,1,1,1, \cdots]^{\top}=[\cdots, 1,1,1,1, \cdots]^{\top} .
$$

It is easily seen that this implies $\mathbf{L} \mathbf{u}_{1}=\mathbf{u}_{1}$ and $\mathbf{H u} \mathbf{u}_{1}=\mathbf{0}$ where $\mathbf{H}$ is the band-Toeplitz matrix corresponding to the highpass analysis. It means that $[\cdots, 1,1,1,1, \cdots]$ is preserved by the lowpass branch and cancelled by the highpass.

However, most of the multiwavelets constructed so far do not even verify this simple requirement, as illustrated in Fig. 5. A solution proposed in [13] and generalized in [4] and [15] is to add some pre/post filtering of the input/output signal to adapt it to the spectral imbalance of the filter bank.

\section{A. Critical Sampling}

A natural way of prefiltering is to partition the input signal into vectors chunks of size 2 and apply on the sequence of vectors the refinement mask $\mathbf{A}(z):=\sum_{k} \mathbf{A}[k] z^{-k}$, where $\mathbf{A}[k]$ are $2 \times 2$ matrices. Thus, we get an input sequence of vectors adapted to the spectral imbalance of the multifilter bank. In that case, we can maintain critical sampling, with the only restriction that the input 

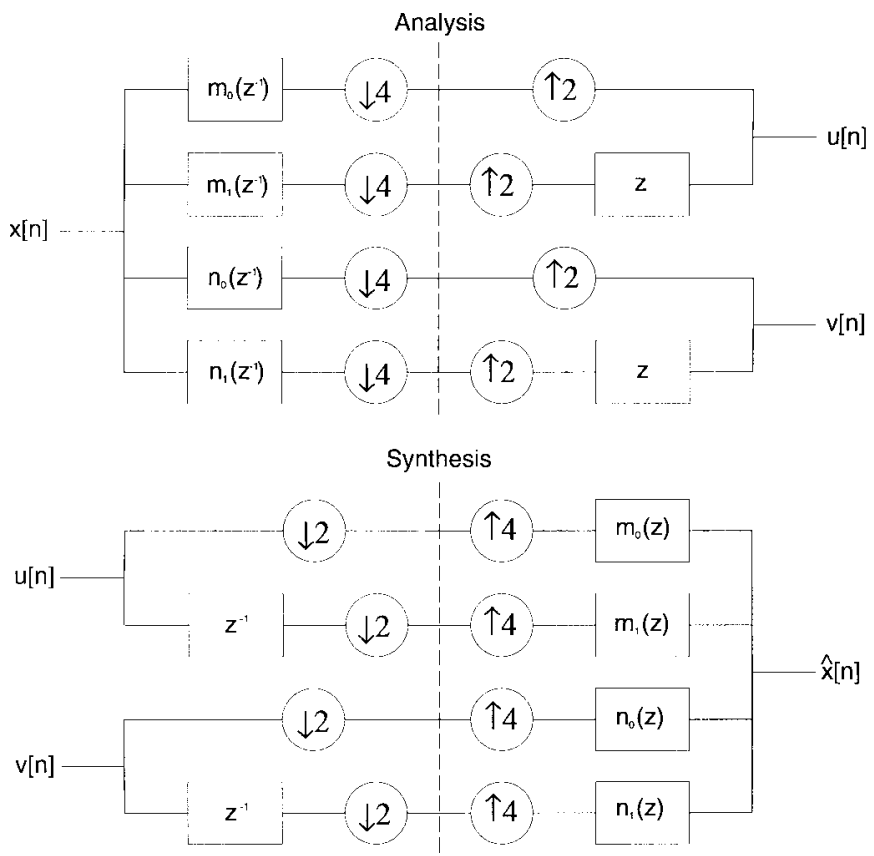

Fig. 3. Multiwavelet filter bank seen as a time-varying filter bank.

signal must be of size $2^{K}$ for some $K$. The reconstruction is easily processed by applying the refinement mask $\mathbf{B}(z)$ inverse of $\mathbf{A}(z)$ onto the output signal (Fig. 2). A simple way of understanding prefiltering is then to see it as a transform such that the input signal $[\cdots, 1,1,1,1, \cdots]$ is mapped to some genuine vector eigensignal associated with the eigenvalue 1 of $\mathbf{L}^{\top}$. For example, with the DGHM multiwavelet [3], we have

$$
\mathbf{L}^{\top}[\cdots, \sqrt{2}, 1, \sqrt{2}, 1, \cdots]^{\top}=[\cdots, \sqrt{2}, 1, \sqrt{2}, 1, \cdots]^{\top} .
$$

The results obtained (Fig. 4) using this trick are of the same order as the ones obtained using a plain Daubechies filter bank with four taps. However, the prefilters constructed so far are destroying either the orthogonality [15] or the linear phase [4] of the system, thus reducing the interest of multiwavelet-based filter banks against usual biorthogonal wavelet-based filter banks.

\section{B. Noncritical Sampling}

Another way of doing pre/post filtering is to allow noncritical sampling and to construct some projection of the input signal on $V_{0}$. For example, with the DGHM multiwavelet, when starting from an input signal $\left[x[0], \cdots, x\left[2^{K}\right]\right]$, we transform it into some redundant vector-valued input sequence

$$
\left[x[0]\left[\begin{array}{c}
\sqrt{2} \\
1
\end{array}\right], \cdots, x\left[2^{K}\right]\left[\begin{array}{c}
\sqrt{2} \\
1
\end{array}\right]\right]
$$

This preprocessing is often called the repeated signal approach. It doubles the size of the input signal but allows us to maintain the orthogonality and linear phase of the system. However, by the redundancy it creates, one cannot use this approach in the framework of signal compression.

As mentioned in [15] and [16], an issue of prefiltering is then to maintain orthogonality, linear phase, and critical sampling at the same time. However, one may rather directly design orthogonal multiwavelets with good balance between the two scaling functions.

\section{BALANCING}

In [8], we gave the definition of a first-order balanced multiwavelet. We impose the condition (13) that the lowpass synthesis operator $\mathbf{L}^{\top}$ of the time-varying filter bank based on this multiwavelet preserve the eigensignal $[\cdots, 1,1,1,1, \cdots]$. One then proves the following theorem.

Theorem: The following conditions are equivalent.

B0) $\mathbf{L}^{\top} \mathbf{u}_{1}=\mathbf{u}_{1}$.

B1) $[1,1]$ is a left eigenvector of $\mathbf{M}(1)$ for $\lambda_{0}(1)=1$.

B2) $\Phi(0)=[1,1]^{\top}$.

B3) $m_{0}(z)+m_{1}(z)$ has zeros ${ }^{1}$ on the unit circle at $j,-1,-j$.

Proof: $[\mathrm{B} 0 \Rightarrow \mathrm{B} 1]$ : Assuming $\mathrm{B} 0$, we have $\mathbf{u}_{1}^{\top} \mathbf{L}=\mathbf{u}_{1}^{\top}$ so that we trivially get condition B1. [B1 $\Rightarrow$ B2]: From (3) at $\omega=0$, using (5) and necessary condition 2), we get that $\Phi(0)$ is a left and right eigenvector associated to the eigenvalue 1 of $\mathbf{M}(1)$; hence, we have the result. [B2 $\Rightarrow \mathrm{B} 3]$ : $\Phi(0)$ is a left and right eigenvector associated with the eigenvalue 1 of $\mathbf{M}(1)$, and from condition 2) in the convergence of the iterated matrix product, we get $\Phi^{\top}(0) \mathbf{M}(-1)=\mathbf{0}$. Since $\Phi(0)=[1,1]^{\top}$, we get that $m_{0}(z)+m_{1}(z)=[1,1] \mathbf{M}\left(z^{2}\right)\left[1, z^{-1}\right]^{\top}$ has roots at $z=$ $j,-1,-j$. [B3 $\Rightarrow$ B0]: Finally, taking $u[n]=[\cdots, 1,1,1,1, \cdots]$ and $v[n]=[\cdots, 0,0,0,0, \cdots]$ in Fig. 3 , we get that the four possible outputs $\hat{x}[4 n+p]=\sum_{k} m_{0}[4 k+p]+m_{1}[4 k+p]$ for $p=0,1,2,3$ are equal [12]. Therefore, $[\cdots, 1,1,1,1, \cdots]$ is an eigensignal of the operator $\mathbf{L}^{\top}$. Thus, we have the equivalence of $\mathrm{B} 0, \mathrm{~B} 1, \mathrm{~B} 2$, and $\mathrm{B} 3$.

We also remark that condition B2 implies that $\phi_{0}(t), \phi_{1}(t)$ are bona-fide scaling functions, i.e., that the initialization rule on which the Mallat algorithm is based apply:

$$
\int x(t) \phi_{i}(t-n) d t \cong x(n)
$$

For $p$-order balancing, we impose the condition that the lowpass branch of a time-varying filter bank based on this multiwavelet preserves the sampled versions of polynomials of degree up to $p-1$. For degree 2 , it means that $[\cdots,-1,0,1,2, \cdots]$ has also to be preserved by $\mathbf{L}^{\top}$.

\section{A. Direct Construction}

A simple way to construct balanced multiwavelets of arbitrary order is to derive them from the complex Daubechies filters [6], [10]. Daubechies filters are constructed using the halfband filter

$$
P(z):=c\left(1+z^{-1}\right)^{N}(1+z)^{N} R(z)
$$

such that $P(z)+P(-z)=1$ with $R\left(e^{j \omega}\right) \geq 0$ and $R\left(e^{j \omega}\right)=$ $R\left(e^{-j \omega}\right)$. One gets the usual Daubechies lowpass filters $D_{N}(z):=$ $\left(1+z^{-1}\right)^{N} B(z)$, where $B(z)$ is a spectral factor of $R(z)$ with real coefficients. We cannot achieve orthogonality and symmetry with real coefficients; however, by allowing complex coefficients in the spectral factorization, one can construct symmetric, orthogonal FIR filters. Writing $[a[0], \cdots, a[N], a[N], \cdots, a[0]]$ for the lowpass filter, we construct the matrix coefficients

$$
\mathbf{A}[i]:=\left[\begin{array}{cc}
\operatorname{Im} a[i] & \operatorname{Re} a[i] \\
\operatorname{Re} a[i] & \operatorname{Im} a[i]
\end{array}\right]
$$

and the refinement mask is then

$$
\mathbf{M}(z):=\frac{1}{2}\left\{\sum_{k=0}^{N} \mathbf{A}[k] z^{-k}+z^{-(N+1)} \sum_{k=0}^{N} \mathbf{A}[N-k] z^{-k}\right\} .
$$

\footnotetext{
${ }^{1}$ Condition B3 was first given by Selesnick in [12].
} 


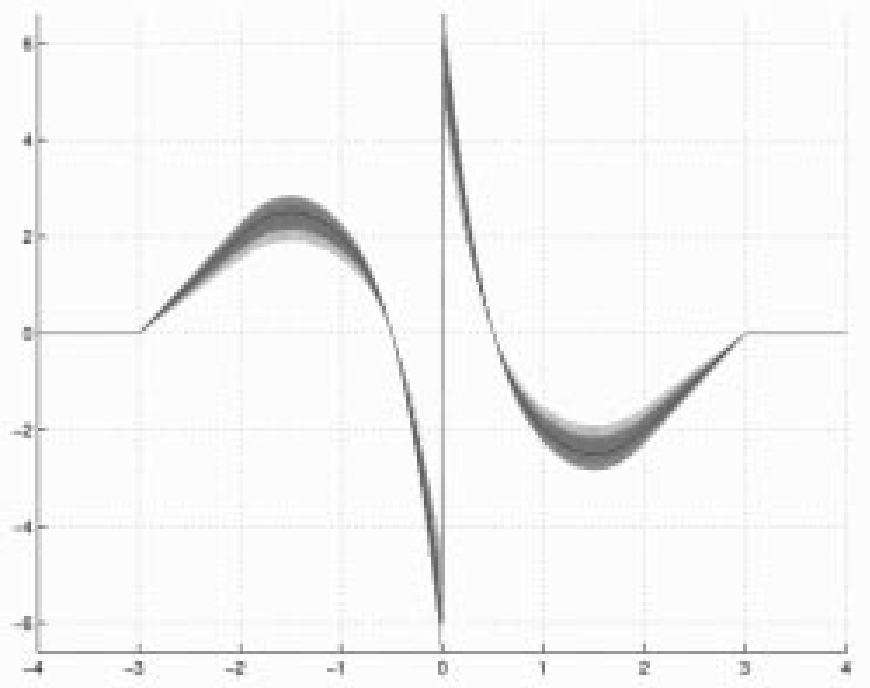

(a)

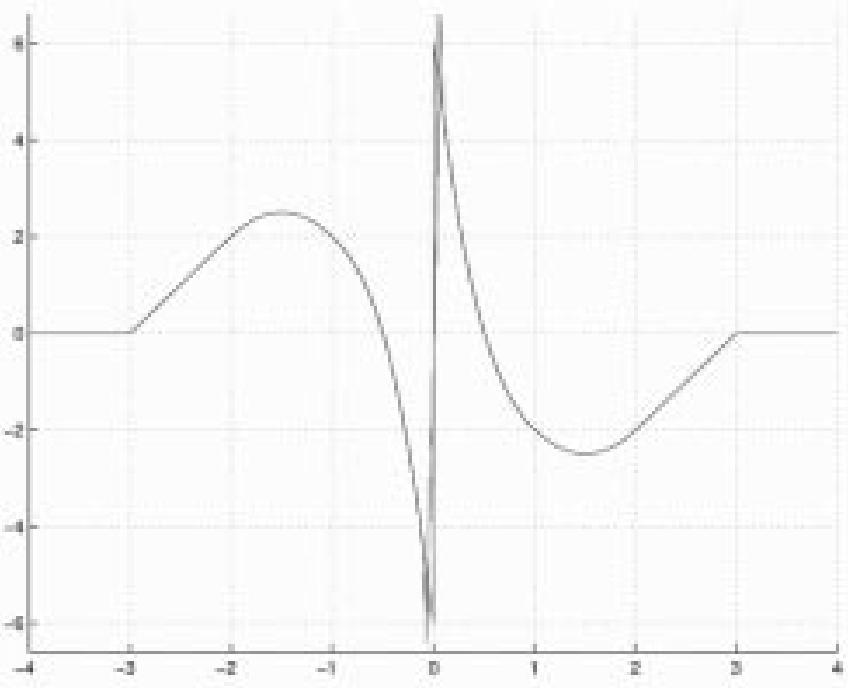

(b)

Fig. 4. Truncation of the first-order detail subband in a DGHM multiwavelet filter bank (i.e., we force $v[n]=0$ in the time-varying implementation). The input signal is symmetric and composed of a constant, a linear, a quadratic, and a cubic parts with a step. (a) Poor robustness of a system based on DGHM without pre/post filtering. (b) Results are greatly improved by using pre/post filtering.

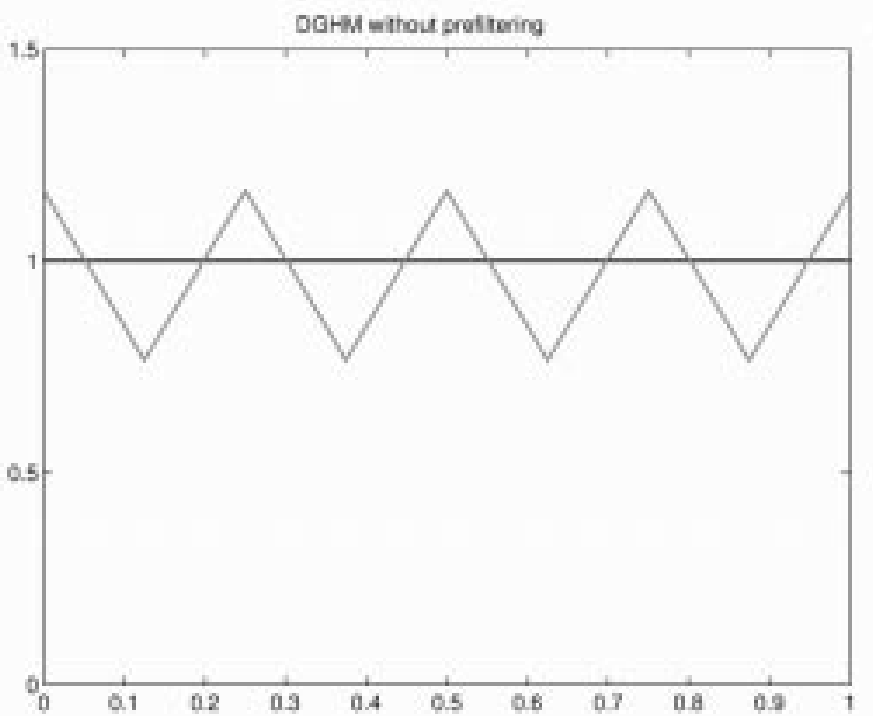

(a)

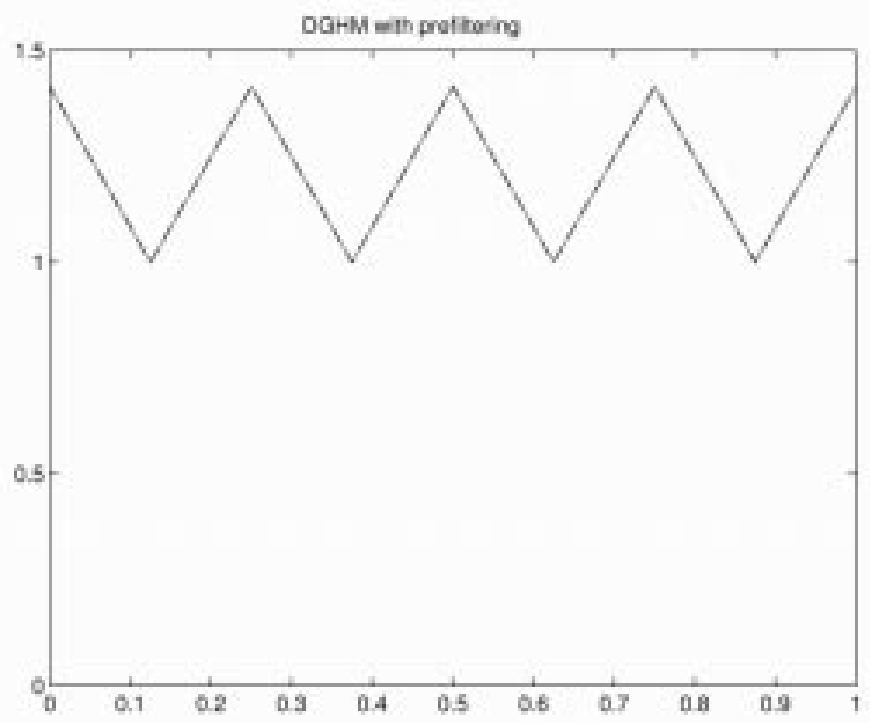

(b)

Fig. 5. (a) Reproduction of the input signal $[\cdots, 1,1,1,1, \ldots]$ by a DGHM multiwavelet-based filter bank without prefiltering. (b) Reproduction of the eigensignal $[\cdots, \sqrt{2}, 1, \sqrt{2}, 1, \cdots]$ by the same system. The mapping of $[\cdots, 1,1,1,1, \cdots]$ to this eigensignal is the key idea of prefiltering.

The multifilter bank is clearly orthogonal. Moreover, defining that $\phi$ has approximation power of $m$ if one can exactly decompose polynomials up to degree $m-1$ using only $\phi_{0}(t), \phi_{1}(t)$ and their integer translates and that $\phi$ has smoothness $p$ if $\phi_{0}(t), \phi_{1}(t)$ have $p$ continuous derivatives, then it is easily seen that the smoothness and approximation power of the Daubechies complex scaling functions and wavelets transfer to the multiscaling functions and multiwavelets. Namely, by defining $\varphi(t):=\phi_{1}(t)+j \phi_{0}(t)$, where $\left[\phi_{0}(t), \phi_{1}(t)\right]$ is the multiscaling function associated with $\mathbf{M}(z)$, we get that $\varphi(t)$ verifies the two-scale equation

$$
j \bar{\varphi}(t)=\sum_{k=0}^{N} a[k] \varphi(2 t-k)+\sum_{k=N+1}^{2 N+1} a[2 N+1-k] \varphi(2 t-k)
$$

so that $\varphi(t)$ is a scaling function associated with the complex Daubechies filters; hence, we get the same smoothness and approximation power for the multiscaling functions and the multiwavelets. We also easily derive that the multiscaling functions and multiwavelets are symmetric/antisymmetric, as seen in Fig. 6. However, this refinement mask when iterated does not converge properly because $\mathbf{M}(1)$ has eigenvalues $1,-1$ with left eigenvectors $[1,1]$, [1, $-1]$. We get only constrained [5], i.e., nonuniform convergence of the Mallat algorithm; hence, the poor behavior of these multiwavelets in applications as seen in Fig. 7.

\section{B. Balancing the Nonbalanced}

Another interesting way of constructing balanced multiwavelets is to balance already existing multiwavelets like the ones constructed 


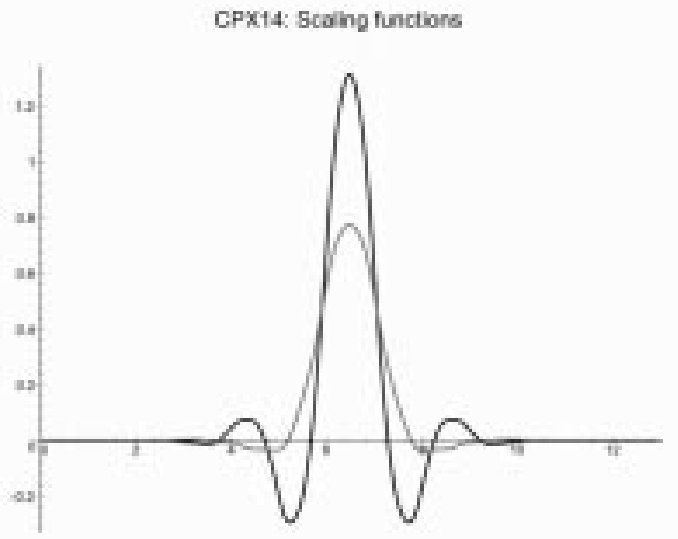

(a)

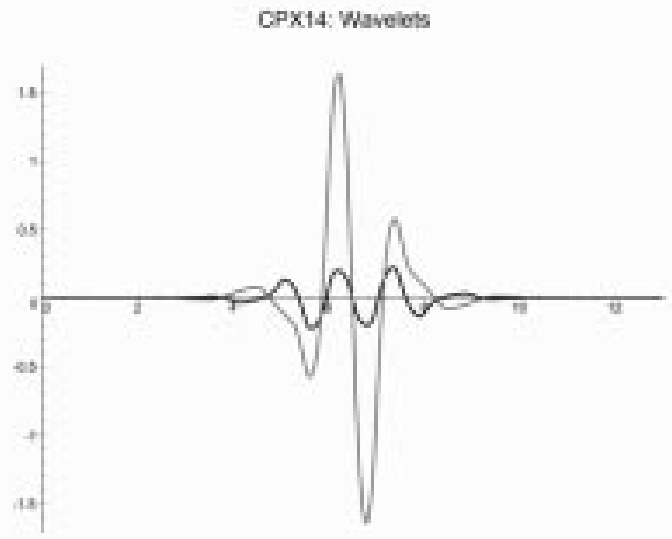

(b)

Fig. 6. Balanced multiwavelet derived from the complex Daubechies filters (same approximation power and smoothness as D14). (a) Scaling functions. (b) Multiwavelets.

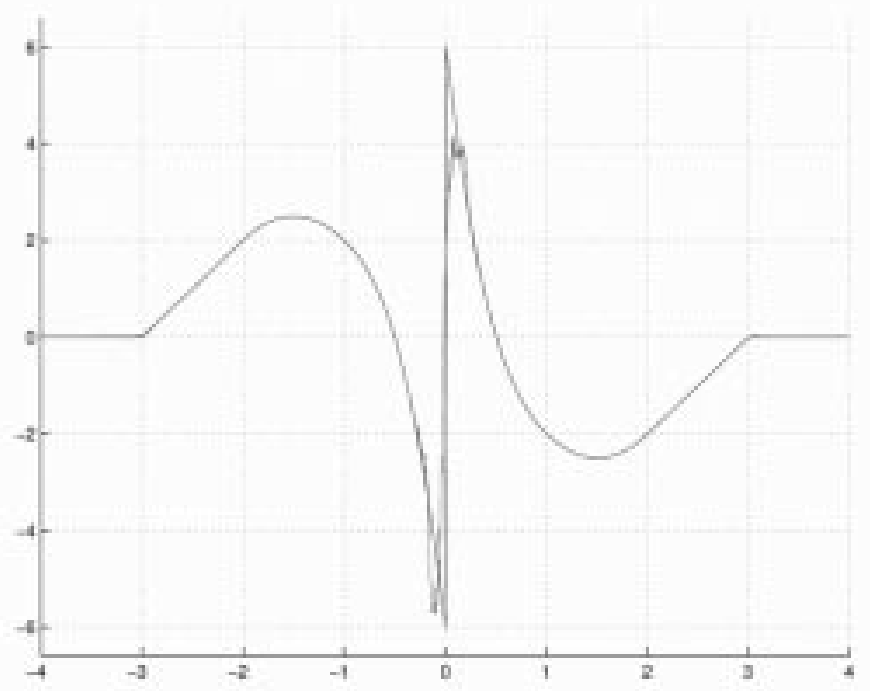

(a)

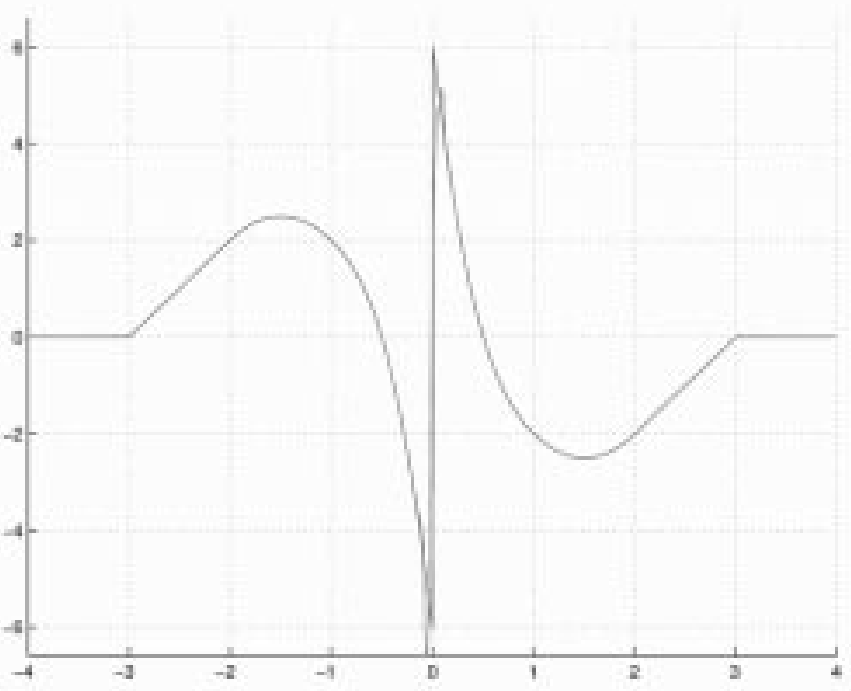

(b)

Fig. 7. Robustness to truncation of the first order detail subband with (a) a $62 \times 2$ taps Daubechies based multiwavelet filter bank and (b) with a Chui based balanced multiwavelet with $82 \times 2$ taps filter bank.

in [1] or [3]. The point is that we want $[1,1]$ to be a left eigenvector associated with eigenvalue 1 of $\mathbf{M}(1)$. The way to achieve this is to use a unitary matrix $R$ such that

$$
[1,1] R^{\top} \mathbf{M}(1) R=[1,1] \text {. }
$$

Defining the new refinement mask

$$
\mathbf{P}(z):=R^{\top} \mathbf{M}(z) R
$$

and the new two-scale equation

$$
\Phi_{P}(2 \omega)=\mathbf{P}\left(e^{j \omega}\right) \Phi_{P}(\omega)
$$

we get that $[1,1]$ is a left eigenvector of $\mathbf{P}(1)$ for $\lambda_{0}(1)=1$ and since the transformation is unitary, $\mathbf{P}$ verifies (5), and therefore, $\Phi_{P}(0)=[1,1]^{\top}$. We notice that in the iteration, $R^{\top}$ and $R$ cancel, except for the first and last term. The convergence of the iterated matrix product (5) for $\mathbf{M}$ imply the convergence for $\mathbf{P}$, and the smoothness and approximation power are also unchanged. Moreover, the whole orthogonality of the filter bank is maintained, and although the symmetry of the scaling functions is usually lost, the symmetry/antisymmetry of the multiwavelets can be maintained by taking for the highpass refinement mask $\mathbf{Q}(z):=\mathbf{N}(z) R$. Namely

$$
\begin{aligned}
\Psi_{P}(\omega) & =\mathbf{N}\left(e^{j(\omega / 2)}\right) R\left\{\prod_{i=2}^{\infty} R^{\top} \mathbf{M}\left[e^{j\left(\omega / 2^{i}\right)}\right] R\right\}\left[\begin{array}{l}
1 \\
1
\end{array}\right] \\
& =\mathbf{N}\left(e^{j \omega / 2}\right) \mathbf{M}_{\infty}(\omega / 2) \Phi_{M}(0)=\Psi_{M}(\omega) .
\end{aligned}
$$

Balancing Chui's multiwavelets [1], we obtained orthogonal, compactly supported multiscaling functions/multiwavelets with symmetric/antisymmetric wavelets (Fig. 8). Moreover, the scaling functions are flipped versions of one another, verify the $[1,1]$ left eigenvector condition, and have the interesting sampling property

$$
\int x(t) \phi_{i}(t-n) d t \cong x\left(n+\frac{i}{2}\right)
$$

These balanced multiwavelets (which are abbrieviated Bat) have shown very good robustness in compression algorithm without any pre/post filtering (Fig. 7). 


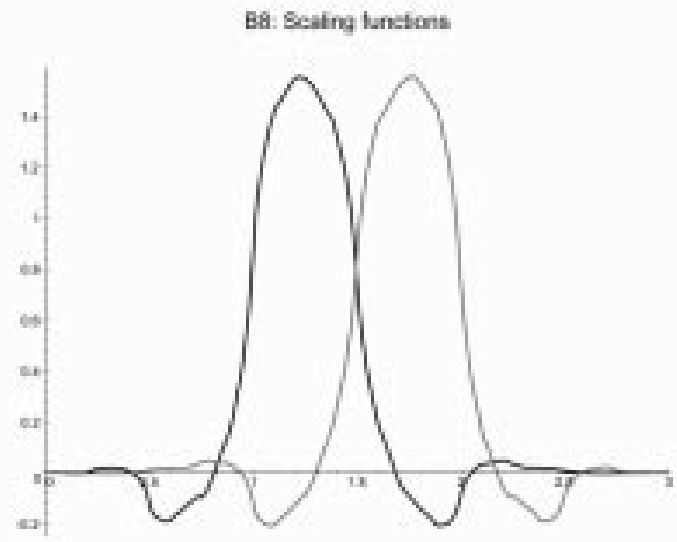

(a)

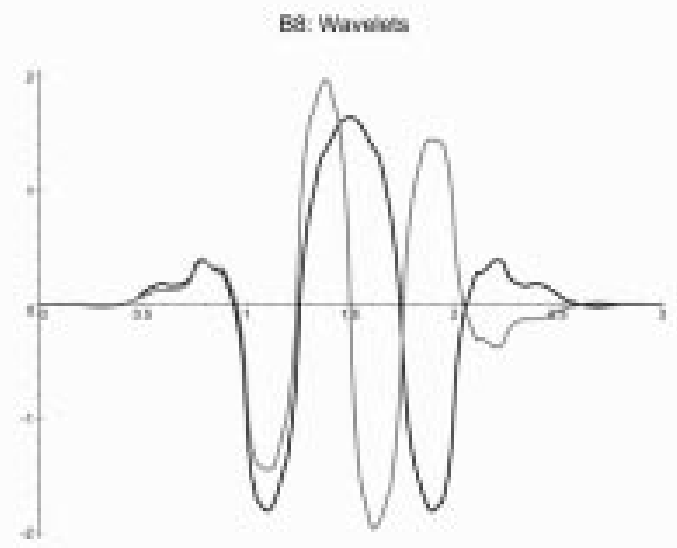

(b)

Fig. 8. Balanced multiwavelet with approximation power of 2. (a) Scaling functions. (b) Multiwavelets. The scaling functions are flipped version of one another. They are orthogonal and have good sampling properties. The wavelets are orthogonal and symmetric/antisymmetric.

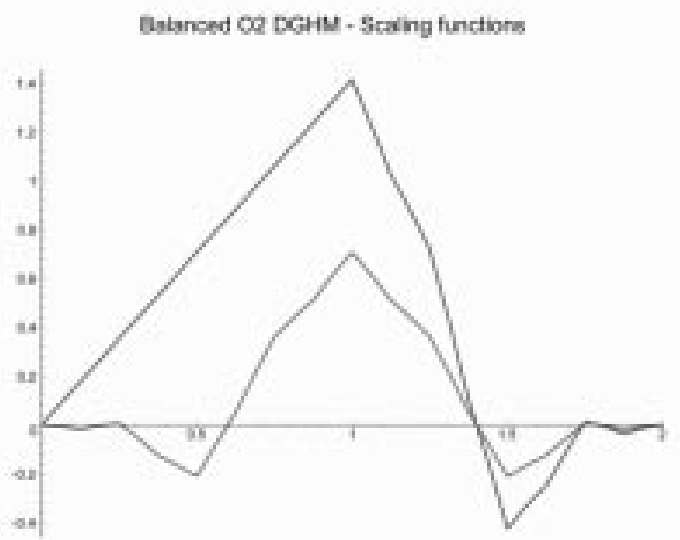

(a)

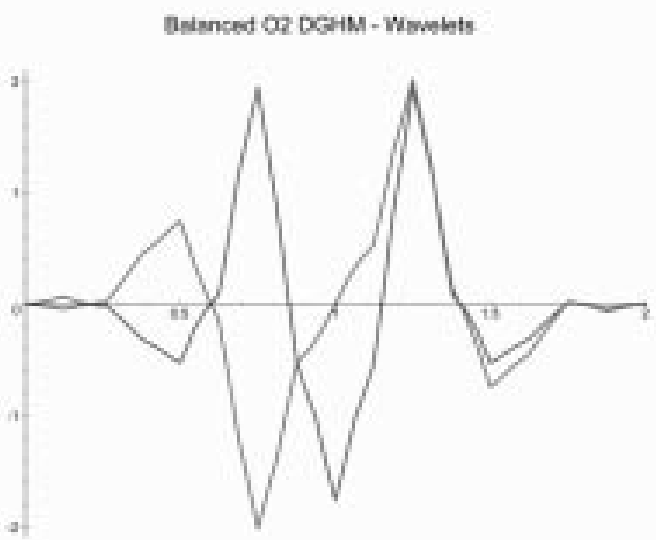

(b)

Fig. 9. Second-order balanced multiwavelet derived from DGHM. Sampled versions of 1 and $t$ are eigensignals of the lowpass branch of the multiwavelet filter bank. (a) Scaling functions. (b) Multiwavelets. The system is still orthogonal but in a weaker sense: the lowpass and highpass branches are mutually orthogonal $(u[n]$ and $v[n]$ are uncorrelated) and the highpass branch is orthogonal (the coefficients $v[n]$ are uncorrelated). Furthermore, the linear phase of the highpass branch is maintained (symmetry/antisymmetry of the wavelets).

\section{Higher Order Balancing}

One can generalize what was previously done for balancing nonbalanced multiwavelets to higher order polynomial input signals. Namely, in the case of DGHM, we have approximation power of 2 , i.e.,

$$
\begin{aligned}
1 & =\sum_{k} \sqrt{2} \phi_{0}(t-k)+\phi_{1}(t-k) \\
t & =\sum_{k} \sqrt{2}\left(k+\frac{1}{2}\right) \phi_{0}(t-k)+(k+1) \phi_{1}(t-k) .
\end{aligned}
$$

Therefore, if we want to preserve the sampled version of 1 and $t$ as input signals, we should transform them into eigensignals of the DGHM based filter bank. Therefore, we get the equations

$$
A\left[\begin{array}{l}
1 \\
1
\end{array}\right]=\left[\begin{array}{c}
\sqrt{2} \\
1
\end{array}\right], \quad A\left[\begin{array}{c}
n \\
n+\frac{1}{2}
\end{array}\right]=\left[\begin{array}{c}
\sqrt{2}\left(n+\frac{1}{2}\right) \\
n+1
\end{array}\right]
$$

leading to

$$
A=\left[\begin{array}{rc}
0 & \sqrt{2} \\
-1 & 2
\end{array}\right]
$$

Defining the new refinement mask

$$
\mathbf{P}(z):=A^{\mathrm{T}} \mathbf{M}(z) A^{-\mathrm{T}}
$$

and the new two-scale equation

$$
\Phi_{P}(2 \omega)=\mathbf{P}\left(e^{j \omega}\right) \Phi_{P}(\omega)
$$

the time-varying filter bank based on this refinement mask keeps constant and linear input signals unchanged. Again, the convergence of the matrix product for $\mathbf{M}$ implies the convergence for $\mathbf{P}$, and the smoothness and approximation power are, therefore, unchanged. However, this time, the symmetry and orthogonality by shifts of the scaling functions are lost. Nevertheless, the system remains orthogonal in the sense that the scaling functions are orthogonal to the wavelets; therefore, it still decorrelates coarse resolution and details. Moreover, as seen in Fig. 9, the symmetry/antisymmetry and orthogonality by shifts of the multiwavelets can be maintained by taking for the highpass refinement mask $\mathbf{Q}(z):=\mathbf{N}(z) A^{-\mathrm{T}}$.

\section{CONCLUSION}

After recalling some basic facts about multiwavelets, we reviewed some of the problems that appear when applying multiwavelets in signal processing. We proposed a new way to solve these problems: the balanced multiwavelets. By imposing the balancing conditions, we have been able to construct robust multiwavelet filter banks for 
processing 1-D signals in a simple way. Thus, we obtained orthogonal (sometimes in a weaker sense), linear-phase FIR systems preserving sampled versions of polynomials. We have now new tools to process images and sounds in an orthogonal linear-phase FIR environment available for further experiments. Some issues remain open, however. We still have to develop some systematic and simple way to construct orthogonal symmetric balanced multiwavelets with any desired approximation power and order of balancing (preservation of sampled versions of higher order polynomial). Since the submission of this correspondence, important new results in that direction have been obtained [9]. We have linked the concept of high-order balancing to a very natural factorization of the lowpass refinement mask that is the counterpart of the well-known zeros at $\pi$ condition for wavelets. This enabled us to clarify the subtle relations between approximation power, smoothness, and balancing order. Using these new results, we have been able to construct a new family of orthogonal multiwavelets with symmetries and compact support that is indexed by the order of balancing. More details (filters coefficients, drawings of the whole family, frequency responses, ...) can be obtained on the World Wide Web at http://lcavwww.epfl.ch/ lebrun.

\section{ACKNOWLEDGMENT}

The authors would like to thank X.-G. Xia, I. Selesnick, and P. Rieder for providing them with their preprints and I. Daubechies, G. Strang, J.-M. Lina, and the reviewers for suggesting very useful remarks and ideas.

\section{REFERENCES}

[1] C. K. Chui and J.-A. Lian, "A study of orthonormal multi-wavelets," Appl. Numer. Math., vol. 20, no. 3, pp. 273-298, 1996.

[2] A. Cohen, I. Daubechies, and G. Plonka, "Regularity of refinable function vectors," J. Fourier Anal. Appl., vol. 3, no. 3, pp. 295-324, 1997.

[3] J. S. Geronimo, D. P. Hardin, and P. R. Massopust, "Fractal functions and wavelet expansions based on several scaling functions," J. Approx. Theory, vol. 78, no. 3, Sept. 1994.

[4] D. P. Hardin and D. W. Roach, "Multiwavelets prefilters I: Orthogonal prefilters preserving approximation order $p \leq 2$," preprint, 1997.

[5] C. Heil and D. Colella, "Matrix refinement equations: Existence and uniqueness," J. Fourier Anal. Appl., vol. 2, no. 4, 1996.

[6] W. Lawton, "Applications of complex valued wavelet transforms to subband decomposition," IEEE Trans. Signal Processing, vol. 41, pp. 3566-3568, Dec. 1993.

[7] W. Lawton, S. L. Lee, and Z. Shen, "An algorithm for matrix extension and wavelet construction," Math. Comput., vol. 65, pp. 723-737, 1996.

[8] J. Lebrun and M. Vetterli, "Balanced multiwavelets," in Proc. IEEE ICASSP, Munich, Germany, 1997.

[9] , "High order balanced multiwavelets," in Proc. IEEE ICASSP, Seattle, WA, 1998.

[10] J.-M. Lina and M. Mayrand, "Complex Daubechies wavelets," Appl. Comput. Harmonic Anal., vol. 2, pp. 219-229, 1995.

[11] P. Rieder and J. A. Nossek, "Multiwavelets transforms based on several scaling functions: Design and implementation," submitted for publication.

[12] I. W. Selesnick, "Multiwavelet bases with extra approximation properties," preprint, 1997.

[13] V. Strela, P. Heller, G. Strang, P. Topiwala, and C. Heil, "The application of multiwavelet filter banks to signal and image processing," IEEE Trans. Image Processing, 1996.

[14] M. Vetterli and G. Strang, "Time-varying filter banks and multiwavelets," in Proc. 6th IEEE Dig. Signal Process. Workshop, Yosemite, CA, 1994.

[15] X.-G. Xia, J. S. Geronimo, D. P. Hardin, and B. W. Suter, "Design of prefilters for discrete multiwavelet transforms," IEEE Trans. Signal Processing, vol. 44, pp. 25-35, Jan. 1996.
[16] X.-G. Xia, "A new prefilter design for discrete multiwavelet transforms," preprint, 1996.

\section{On the Least Asymmetric Wavelets}

\author{
Miloš I. Doroslovački
}

Abstract - The asymmetry of Daubechies' scaling functions and wavelets can be diminished by minimizing a special second moment in time for the wavelet-generating discrete-time filter. The moment is involved in an uncertainty relation for discrete-time signals. Other measures of asymmetry are addressed as well, and corresponding results are compared.

\section{INTRODUCTION}

Daubechies' wavelets are continuous-time functions whose mother wavelet has a finite support width. They constitute an orthonormal basis for the functions of finite energy [1], [2]. Moreover, the mother wavelet $w(\tau)$ has the highest number of vanishing moments for a given support width, where $N$ vanishing moments mean

$$
\int_{-\infty}^{+\infty} \tau^{i} w(\tau) d \tau=0
$$

for $i=0,1, \ldots, N-1$. The corresponding scaling functions are orthonormal within the same scale and orthogonal to wavelets of the same and lower scales. The basic scaling function is defined [2] as one having the spectrum

$$
V(\omega)=\prod_{i=1}^{+\infty} \frac{1}{\sqrt{2}} G\left(\frac{\omega}{2^{i}}\right) .
$$

For the mother wavelet, we have [2]

$$
W(\omega)=\frac{1}{\sqrt{2}} H\left(\frac{\omega}{2}\right) \prod_{i=2}^{+\infty} \frac{1}{\sqrt{2}} G\left(\frac{\omega}{2^{i}}\right) .
$$

$G(\omega)$ and $H(\omega)$ are transfer functions of special finite impulse response digital filters. First, they are conjugate quadrature and power complementary filters [3], [4], i.e., $H(\omega)=-G^{*}(\omega+\pi) e^{-j(2 L+1) \omega}$, and $|G(\omega)|^{2}+|G(\omega+\pi)|^{2}=2$. Second, in order to satisfy the vanishing moment requirement, $G(\omega)$ has the form [2]

$$
G(\omega)=\sqrt{2}\left(\frac{1+e^{-j \omega}}{2}\right)^{N} P(\omega)
$$

where

$$
|P(\omega)|^{2}=\sum_{k=0}^{N-1}\left(\begin{array}{c}
N-1+k \\
k
\end{array}\right)\left(\frac{1-\cos \omega}{2}\right)^{k} .
$$

The impulse response $g(t)$ has $2 N$ coefficients. It can be chosen to be causal [5] and to start at $t=0$. In that case, we can choose $L=N-1$ and obtain that $h(t)$ is also causal and starts at $t=0$, i.e., we have $h(t)=(-1)^{t} g^{*}(2 N-1-t)$.

Manuscript received February 15, 1997; revised November 30, 1997. The associate editor coordinating the review of this paper and approving it for publication was Prof. Ali N. Akansu.

The author is with the Department of Electrical Engineering and Computer Science, George Washington University, Washington, DC 20052 USA (e-mail: doroslov@ seas.gwu.edu).

Publisher Item Identifier S 1053-587X(98)02553-7. 